Basic nutritional investigation

\title{
Olive polyphenol effects in a mouse model of chronic ethanol addiction
}

\author{
Valentina Carito Ph.D. a, Mauro Ceccanti Ph.D. ${ }^{\mathrm{b}}$, Vincenzo Cestari Ph.D. ${ }^{\mathrm{a}}$, \\ Fausta Natella Ph.D. ${ }^{\text {, }}$, Cristiano Bello Ph.D. ${ }^{\text {c }}$, Roberto Coccurello Ph.D. ${ }^{\text {a, }}$, \\ Rosanna Mancinelli Ph.D. ${ }^{\mathrm{d}}$, Marco Fiore Ph.D. ${ }^{\mathrm{a}, *}$ \\ a Institute of Cell Biology and Neurobiology, CNR; IRCCS S. Lucia Foundation, Italy \\ ${ }^{\mathrm{b}}$ CRARL Lazio, Sapienza University, Rome, Italy \\ ${ }^{\mathrm{c}}$ Entecra, Rome, Italy \\ ${ }^{\mathrm{d}}$ Centro Nazionale Sostanze Chimiche, ISS, Rome, Italy
}

\section{A R T I C L E I N F O}

Article history:

Received 24 May 2016

Accepted 28 August 2016

\section{Keywords:}

Alcoholism

Mouse

Oxidative stress

Olive polyphenols

\begin{abstract}
A B S T R A C T
Objectives: Alcohol addiction elicits oxidative imbalance and it is well known that polyphenols possess antioxidant properties. We investigated whether or not polyphenols could confer a protective potential against alcohol-induced oxidative stress.

Methods: We administered (per os) for two months $20 \mathrm{mg} / \mathrm{kg}$ of olive polyphenols containing mostly hydroxytyrosol in alcoholic adult male mice. Hydroxytyrosol metabolites as hydroxytyrosol sulfate 1 and hydroxytyrosol sulfate 2 were found in the serum of mice administered with polyphenols with the highest amount in animals treated with both polyphenols and alcohol. Oxidative stress was evaluated by FORT (free oxygen radical test) and FORD (free oxygen radical defense) tests.

Results: Alcoholic mice showed a worse oxidative status than nonalcoholic mice (higher FORT and lower FORD) but polyphenol supplementation partially counteracted the alcohol pro-oxidant effects, as evidenced by FORT.

Conclusions: A better understanding of the antioxidant protection provided by polyphenols might be of primary interest for drug discovery and dietary-based prevention of the damage associated with chronic alcohol abuse.
\end{abstract}

(c) 2016 Elsevier Inc. All rights reserved.

\section{Introduction}

In recent years, considerable attention has been devoted to lifestyle and diet as key strategies to improve health and to fight chronic diseases. As for nutrition and diet, plant polyphenols, which are bioactive molecules present in our diet, have been the subject of extensive investigation [1]. Polyphenols are a class of organic compounds characterized by the presence of multiple phenolic units. Among the most important polyphenols tannins are found in all families of vegetables, comprising up to $50 \%$ of the dry weight of leaves. There are a number of food sources of polyphenols, such as fruits and vegetables, red wine, green and black tea, coffee, chocolate, olive leaves, extra virgin olive oil, and olives [2] and they are well known to elicit crucial biological

\footnotetext{
* Corresponding author. Tel.: 3906 501703239; fax: 3906501703313.

E-mail address: marco.fiore@cnr.it (M. Fiore).
}

effects including antioxidant, anticancer and antiinflammatory properties [3]. Indeed, animal models and clinical studies indicate that polyphenols may have protective effects in various pathologic states including inflammation, apoptosis, mitochondrial dysfunction and, particularly relevant, oxidative stress and reactive oxygen species (ROS) production [4]. In this regard olive oil is considered an important constituent of the traditional Mediterranean diet and many of the health advantages of this diet have been attributed to olive oil's polyphenols [5,6].

Oxidative imbalance is one of the most important mechanisms leading to alcohol-induced toxicity. The metabolism of ethanol is closely linked to ROS generation and oxidative stress. An important area of research in the study of alcohol and alcoholism is the understanding of the role of ROS in ethanol-induced disease for its importance in the development of innovative therapeutics to prevent ROS action and the toxic effects of ethanol [7]. 
Alcohol induces oxidative stress by direct action, increasing the production of oxidant chemical species, and/or by indirect action, reducing the antioxidant capacity of the cells. The consequences are alterations of lipids and proteins and epigenetic modifications of DNA that is particularly vulnerable to the effects of oxidative stress.

Occasional exposure to alcohol is sufficient to induce an imbalance in the intracellular redox state and produce tissue damage. Some target tissues of ethanol intoxication are the brain and the liver, which show a high metabolic rate involving a large amount of oxygen consumption mainly required to regenerate the adenosine triphosphate (ATP) necessary to maintain the intracellular ion homeostasis and to allow cell survival [8]. However, the elevated oxygen consumption makes the tissue more susceptible to generate oxidative stress and ROS hyperproduction [8].

In this study, we examined the causal relationship between polyphenols supplementation and oxidative stress in a mouse model of chronic alcohol addiction, which is known to be characterized by oxidative imbalance. We aimed at investigating whether or not polyphenols may confer a protective potential against alcohol-associated damage by providing antioxidant defense in mice.

\section{Methods}

Animals

CD-1 outbred male mice were used in this experiment. All animals were 3 mo old and housed in groups of 5 mice at the beginning of the experiments in Plexiglas cages $(33 \times 13 \times 14 \mathrm{~cm})$ under standardized conditions with pellet food (enriched standard diet purchased from Mucedola, Settimo Milanese, Italy). Food (Purina Lab Chow \# 5015) and water were available ad libitum. A 12 L:12 D lighting regime was used.

40 male CD-1 mice were randomly divided into four groups: (i) a group of mice $(n=10)$ received sucrose dissolved in water at equivalent caloric intake of the ethanol group and was used as control group; (ii) another group of mice $(\mathrm{n}=10)$ received ad libitum, as only source of liquid, after an habituation period, ethanol $11 \%$ dissolved in water for $90 \mathrm{~d}$; (iii) a third group of mice $(\mathrm{n}=10)$ received polyphenols ( $20 \mathrm{mg} \bullet \mathrm{kg}^{-1} \bullet \mathrm{d}^{-1}$ ) dissolved in sucrose; and (iv) a further group of mice $(n=10)$ received polyphenols $\left(20 \mathrm{mg} \bullet \mathrm{kg}^{-1} \bullet \mathrm{d}^{-1}\right)$ dissolved in ethanol 11\% (again following an habituation period). The four groups are named, respectively: (i) $\mathrm{Ctrl}$; (ii) $\mathrm{EtOH}$; (iii) Poly; and (iv) $\mathrm{EtOH}+$ Poly. Ethanol used for the preparation of the drinking solution was obtained from Sigma-Aldrich (St. Louis, Missouri, USA) and was of analytical grade. Fluid intake was measured regularly and the amounts consumed were calculated [9]. All groups received pellet food ad libitum as above. Food intake was measured regularly and the daily amounts consumed were calculated. Two months after treatment, mice were used for the experiments. All efforts were made to minimize and reduce animal suffering and for limiting the number of animals used. All animal experiments were carried out following the procedure described by the guidelines of the European Community Council Directive of 24 November 1986 (86/609/EEC). Permission number 08-2014 of February 3, 2014.

\section{Polyphenol's blend}

The polyphenols used in the present study, as indicated by the manufacturer (www.phenopharm.it), derive from a natural standardized olive pulp (Olea europaea L.) extract obtained by an eco-sustainable patented mechanical process (Table 1). It is a by-product of the olive residues (pomace) obtained following olive pressing during the production of extra virgin olive oil. Specifications of the mixture, as indicated by the manufacturer, are shown in a previous study [10]. We used a blend of phenolic compounds because it has been proposed that most of the health benefits associated with virgin olive oil are due to its minor components [5]. Due to the possible synergisms, a combination of biophenols is supposed to possess a stronger action for counteracting different stages of oxidative damage than a single compound [11].

\section{Blood samples}

Animals were sacrificed by a guillotine 1 wk after the behavioral experiments. The blood was collected in vials and quickly centrifuged at $10000 \mathrm{rpm}$ for $15 \mathrm{~min}$ for serum preparation ( $\mathrm{n}=5$ per group) or in heparin vials
Table 1

Phenolea Active Complex Composition

\begin{tabular}{|c|c|}
\hline \multicolumn{2}{|l|}{$\begin{array}{l}\text { Specification of Phenolea_Active Complex } \\
\text { as indicated by the manufacturer }\end{array}$} \\
\hline Appearance & Red rubin molasses \\
\hline Solubility in water, \% & $>99$ \\
\hline \multicolumn{2}{|l|}{ Microbiologic } \\
\hline Salmonellae SPP & absent in $25 \mathrm{~g}$ \\
\hline Escherichia coli & absent in $1 \mathrm{~g}$ \\
\hline Yeast and molds, CFU/g & $\leq 5 \times 102$ \\
\hline Enterobacteria, CFU/g & $\leq 1 \times 102$ \\
\hline Total plate count, CFU/g & $\leq 5 \times 105$ \\
\hline Pesticides & absent \\
\hline Aflatoxins & absent \\
\hline Ochratoxin & absent \\
\hline Polycyclic aromatic hydrocarbons mg/kg & $<1$ \\
\hline \multicolumn{2}{|l|}{ Chemical composition of Phenolea_Active Complex } \\
\hline Moisture & $28 \%$ \\
\hline Carbohydrates & $61 \%$ \\
\hline Ashes & $6.5 \%$ \\
\hline Proteins & $2.5 \%$ \\
\hline Fats & $0 \%$ \\
\hline Crude fiber & $2 \%$ \\
\hline \multicolumn{2}{|l|}{ Phenolic composition of Phenolea_Active Complex } \\
\hline Total polyphenols & $5 \%$ \\
\hline \multicolumn{2}{|l|}{ Phenolic families (\% on total polyphenols) } \\
\hline Hydroxytyrosol & $30 \%$ \\
\hline Other hydroxytyrosol derivatives & $20 \%$ \\
\hline Ligstroside and derivatives & $6 \%$ \\
\hline Total secoiridoid acids & $14 \%$ \\
\hline Total phenolic acids & $10 \%$ \\
\hline Oleocanthal & $2 \%$ \\
\hline $\begin{array}{l}\text { Other polyphenols (flavonoids, anthocyanins, } \\
\text { oleuropein, lignans thyrosol) }\end{array}$ & $18 \%$ \\
\hline
\end{tabular}

CFU, colony-forming unit; SPP, species

for the measurement of blood ethanol levels ( $n=5$ per group). Serum aliquots were then stored at $-80^{\circ} \mathrm{C}$ while heparin vials were stored at $4{ }^{\circ} \mathrm{C}$.

Blood ethanol levels by gas chromatography/head space procedure

Gas chromatography/Head Space procedure was applied in this research to determine blood alcohol concentration in whole blood samples $(n=5$ for each group). In this research, a Clarus 600 Gas Chromatography Perkin Elmer and a TurboMatrix 40 Trap HeadSpace Sampler Perkin Elmer with FID detector was used. Analytical conditions were set up and the method was validated by a previous study [12]. From each blood sample was collected $100 \mu \mathrm{L}$ of whole blood with a micropipette and transferred this volume into a gas chromatography vial. The gases which are formed inside gas chromatography vial were collected to be analyzed. If the sample was not analyzed in the same day as its collection, it was important to firmly close the vial to prevent the evaporation of ethanol during time and to conserve the vial inside of a refrigerator. Standard solutions were set up for calibration curve at $100 \mathrm{mg}, 50 \mathrm{mg}, 25 \mathrm{mg}, 12.5 \mathrm{mg}, 6.2 \mathrm{mg}$, and $3.1 \mathrm{mg}$ of ethanol and were obtained by consequent dilutions of pure ethanol in distilled water.

Free oxygen radicals defence and free oxygen radicals test

Free oxygen radicals defence (FORD) and free oxygen radicals (FORT) tests were carried out using two specific kits (both purchased by Callegari, Parma, Italy) following the instruction provided by the manufacturer $[13,14]$. Blood serum was used both for the FORT and FORD determination ( $\mathrm{n}=5$ for each group). FORD test allows the determination of free oxygen radical defense. Briefly, this test uses a preformed stable and colored radical and determines the decrease in absorbance that is proportional to the antioxidant concentration of the sample according to Lambert Beer's law [15]. In the presence of an acidic buffer $(\mathrm{pH}=5.2)$ and a suitable oxidant $\left(\mathrm{FeCl}_{3}\right)$ the chromogen, which contains 4-amino- $N, N$-diethylaniline sulfate forms a stable and colored radical cation photometrically detectable at $505 \mathrm{~nm}$. Antioxidant compounds in the sample reduce the radical cation of the chromogen quenching the color and producing a decoloration of the solution, which is proportional to their concentration. The absorbance values obtained for the samples are compared with a standard curve obtained using Trolox (6-hydroxy-2,5,7,8-tetramethylchroman-2-carboxylic acid), a water-soluble analog of vitamin E commonly used as a reference. 
By contrast, FORT test allows the determination of free oxygen radicals (ROSs) through a colorimetric assay based on the ability of transition metals, such as iron, to catalyze the breakdown of hydroperoxides ( $\mathrm{ROOH}$ ) into derivative radicals, according to Fenton's reaction [15]. Briefly, when $20 \mu \mathrm{L}$ of the blood serum sample was dissolved in an acidic buffer, the hydroperoxides reacted with the transition metal ions liberated from the proteins in the acidic medium and were converted to alkoxy- $\left(\mathrm{RO}_{\mathbf{m}}\right)$ and peroxy- (ROO $\left.\mathbf{m}\right)$ radicals. The radical species produced by the reaction interact with an additive (phenylendiamine derivative $\left(2 \mathrm{CrNH}_{2}\right)$ ) that forms a colored, fairly long-lived radical cation evaluable by spectrophotometer at $505 \mathrm{~nm}$ (linear kinetic-based reaction, $37^{\circ} \mathrm{C}$ ). The intensity of the color correlates directly to the quantity of radical compounds and to the hydroperoxide concentration and, consequently, to the oxidative status of the sample according to the Lambert Beer law (Form CR 2000; Callegari, Parma, Italy).

\section{Serum hydroxytyrosol measurement}

Hydroxytyrosol, also known as DOPET, a derivate of dopamine, is naturally present in the mouse serum [5]. Indeed, the serum for hydroxytyrosol and its derivates measurement was immediately prepared by centrifugation at $1500 \mathrm{~g}$ for $30 \mathrm{~min}$ at $20^{\circ} \mathrm{C}$. Before analysis, the aliquots of serum from each animal were thawed and pooled ( 1 aliquot for each treatment). The sample were acidified with formic acid and diluted to $0.8 \mathrm{~mL}$ with milliQ water (water purified using Millipore MilliQ lab water system, Billerica, MA, USA); salicylic acid (20 ng) was added as internal standard. The samples were centrifuged at $13000 \mathrm{rpm}$ for $30 \mathrm{~min}$ at $4^{\circ} \mathrm{C}$, and supernatants were purified by SPE using Oasis HLB $30 \mathrm{mg}$ cartridges (Waters, Milford, MA). The eluted fractions were evaporated by a gentle flow of nitrogen, and the residues were dissolved in $0.2 \mathrm{~mL}$ of methanol, filtered through $0.20 \mu \mathrm{m}$ PVDF filters and injected. Quantitative online HPLC-ESI-MS/MS analyses were performed using HPLC system interfaced to an Applied Biosystems (Foster City, CA, USA) API3200 Q-Trap instrument working with triple quadrupole analyser in Multiple Reaction Monitoring (MRM) mode. LC analyses were conducted using a system equipped with a 200 binary pump (PerkinElmer, Waltham, MA, USA). Samples were injected $(10 \mu \mathrm{L})$ into an Atlantis column T3 (Waters) $(150 \times 2.1 \mathrm{~mm}, 3 \mu \mathrm{m})$ and eluted at flow rate of $0.2 \mathrm{~mL} / \mathrm{min}$. Mobile phase A was $\mathrm{H}_{2} \mathrm{O}$ containing $0.1 \%$ formic acid while mobile phase B was acetonitrile containing $0.1 \%$ formic acid. Elution was carried out using a linear gradient commencing at $0 \% \mathrm{~B}$ and changing to $50 \% \mathrm{~B}$ in $10 \mathrm{~min}$, isocratic $50 \% \mathrm{~B}$ in $5 \mathrm{~min}$, then to $80 \% \mathrm{~B}$ in $1 \mathrm{~min}$, isocratic $80 \% \mathrm{~B}$ for $4 \mathrm{~min}$, and finally to $0 \% \mathrm{~B}$ in $1 \mathrm{~min}$. Column was then equilibrated for $10 \mathrm{~min}$ at $0 \%$ B for $10 \mathrm{~min}$. The column was kept at $30^{\circ} \mathrm{C}$, using a Peltier Column Oven Series 200 (PerkinElmer). The flow from the chromatograph was injected directly into the ESI source. The API 3200 ES source was tuned by infusing solutions of hydroxytyrosol, tyrosol and salicylic acid ( $1 \mathrm{ng} / \mu \mathrm{L}$ in methanol) into the source at a flow rate of $1 \mu \mathrm{L} / \mathrm{min}$. The MS operated with an electrospray voltage at $-4500 \mathrm{~V}$ and with source temperature of $450^{\circ} \mathrm{C}$. Nitrogen was used as ion spray (GS1), dryng (GS2), and curtain gas at 20, 40 and 40 arbitrary units, respectively. The declustering potential (DP), collision energy (CE) and entrance potential (EP) were $-32,-20$, and $-7 \mathrm{~V}$ for hydroxytyrosol; $-32,-20$, and $-7 \mathrm{~V}$ for tyrosol; and $-42,-15$, and $-8 \mathrm{~V}$ for salicylic acid, respectively. Hydroxytyrosol, tyrosol, hydroxytyrosol sulfates, and salicylic acid were detected with MRM transition of 153/123, 137/106, 233/153, and $137 / 93[\mathrm{M}-\mathrm{H}]^{-}$, respectively. Data acquisition and processing were performed using Analyst software 1.5.1. The quantification of phenolic compounds was performed by using a calibration curve; due to lack of standard, the quantification of hydroxytyrosol sulfates was tentatively quantified using the calibration curve of hydroxytyrosol. The chromatographic analysis of the serum revealed two different hydroxytyrosol sulfates (named HTS1 and HTS2), this was probably due to the presence of sulfate group in different positions.

\section{Statistical analysis}

Data were analyzed by analysis of variance (ANOVA) considering as main factors ethanol and polyphenols administration. Post-hoc comparisons were performed using the Tukey's HSD test. Data are illustrated as mean \pm SEM.

\section{Results}

\section{Polyphenols effects on food and liquid intake and body weight}

Under the present experimental conditions, despite having ingested more liquid and food than the other two groups $(\mathrm{F}[3,12]=47.98 ; \mathrm{F}[3,12]=11.74$, respectively; $P<0.005)$, the polyphenols' group did not show significant changes in body weight as shown at the end of the treatment $(\mathrm{F}[3,36]=1.35$;
Table 2

Mouse body weight and mean daily food and liquid consumption

\begin{tabular}{|c|c|c|c|c|c|c|c|c|}
\hline & \multicolumn{2}{|l|}{ Ctrl } & \multicolumn{2}{|l|}{$\mathrm{EtOH}$} & \multicolumn{2}{|l|}{ Poly } & \multicolumn{2}{|c|}{ EtOH+Poly } \\
\hline & Mean & SEM & Mean & SEM & Mean & SEM & Mean & SEM \\
\hline \multicolumn{9}{|l|}{ Mouse body weight } \\
\hline \multicolumn{9}{|c|}{ Day of treatment } \\
\hline 0 & 34.44 & 0.9 & 34.51 & 0.6 & 35.83 & 0.8 & 35.74 & 0.7 \\
\hline 30 & 40.48 & 1.1 & 40.17 & 0.7 & 42.37 & 1.0 & 41.88 & 0.6 \\
\hline 60 & 44.66 & 1.0 & 43.64 & 0.9 & 46.94 & 1.2 & 45.04 & 0.7 \\
\hline 90 & 45.99 & 1.3 & 44.57 & 0.8 & 47.53 & 1.2 & 45.73 & 0.8 \\
\hline \multicolumn{9}{|c|}{ Mean daily food and liquid consumption per cage } \\
\hline $\begin{array}{l}\text { Mean food } \\
\text { consumption }\end{array}$ & 94.41 & 5.1 & 98.97 & 5.4 & 112.2 & 6.0 & 101.38 & 5.5 \\
\hline $\begin{array}{l}\text { Mean liquid } \\
\text { consumption }\end{array}$ & 66.66 & 3.0 & 61.57 & 2.7 & 91.85 & 4.2 & 62.45 & 2.7 \\
\hline
\end{tabular}

SEM, standard error of the mean

$P=0.27$ ) (Table 2). The ethanol blood levels in the ethanol groups ranged from 3.2 to $20.8 \mathrm{mg} / 100 \mathrm{~mL}$.

\section{Oxidative stress evaluation}

In order to evaluate whether polyphenols might exert antioxidant effects in alcoholic mice we used two well established assays, the FORT (free oxygen radical test) and the FORD (free oxygen radical defense) tests [15] (Fig. 1). We found that FORT levels in blood serum were significantly higher in $\mathrm{EtOH}$ mice as compared to controls. Quite interestingly, the presence of polyphenols seems to have partially counteracted this effect $(\mathrm{F}[3,16]=299.95, P<0.01$ in the ANOVA: see Fig. $1 \mathrm{~A}$ for post-hoc comparisons). However, the levels of FORD in blood serum were significantly lower in both group of ethanol-treated mice (EtOH and EtOH+Poly) as compared to Ctrl and Poly animals (F $[3,16]=64.8, P<0.01$ in the ANOVA: see Fig. $1 \mathrm{~B}$ for post-hoc comparisons). Quite interestingly, polyphenols supplementation increased the natural free oxygen radical defense (FORD) as shown by the significant differences between the polyphenols group versus the control group (see post-hoc comparisons of Fig. 1B).

\section{Serum hydroxytyrosol and metabolites}

Table 3 shows the measurement of hydroxytyrosol (HT), tyrosol (T), hydroxytyrosol sulfates (HTS), and hydroxytyrosol glucuronide (HTG) in the serum of each experimental group. In the serum of $\mathrm{Ctrl}$ mice and $\mathrm{EtOH}$ mice, as previously shown [10] low DOPET levels were observed. Both isomers of hydroxytyrosol sulfates (HTS1 and HTS2) were observed in the serum of Poly and Poly + EtOH mice.

\section{Discussion}

This is the first study to demonstrate that olive polyphenols' supplementation in a mouse model of ethanol addiction may confer protection against ethanol-induced oxidative stress by reducing serum free oxygen radicals but not affecting the free oxygen radicals defense. Under the present experimental conditions, our data demonstrate that (i) polyphenols can partially protect from ethanol-induced oxidative stress although further studies analyzing multiple methods of oxidative stress are required; (ii) polyphenols by themselves do not appear to potentiate the natural endogenous antioxidant resources following chronic alcohol exposure; (iii) however, polyphenols administration by themselves may potentiate the natural endogenous antioxidant resources in normal conditions. 

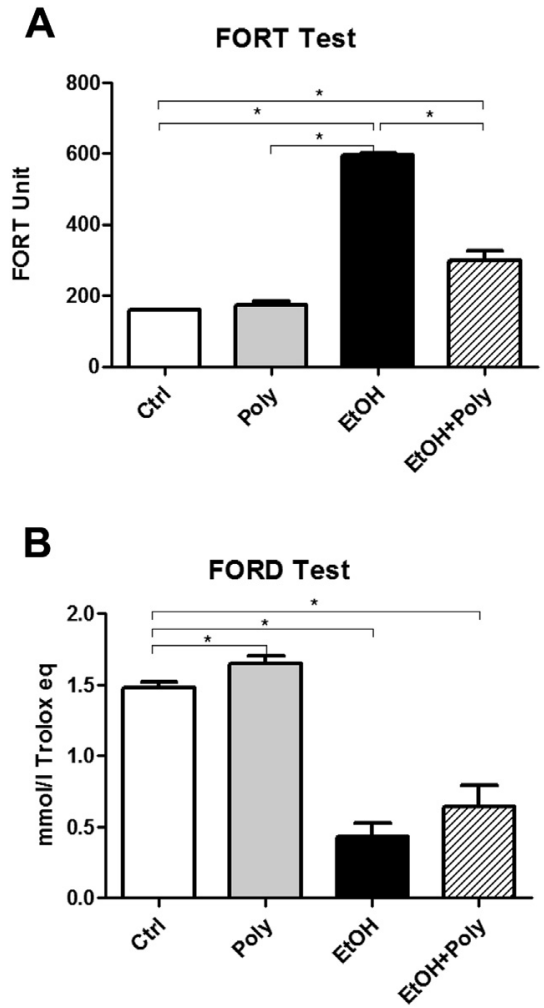

Fig. 1. FORT (A, expressed as FORT unit; see Methods) and FORD levels (B, expressed as mmol/Trolox eq; see Methods) in blood serum of control or alcoholic mice treated or not with olive polyphenols. The vertical lines in the figures indicate pooled standard error means (SEM) derived from appropriate error mean square in the ANOVA. Asterisks indicate significant differences between groups $\left({ }^{*} P<0.05\right)$.

Similar findings were previously observed in aged rats treated with an olive leaf extract [16] showing a decrease in oxidative stress by acting as an antioxidant per se but not affecting the antioxidant system. In this study, we administered a blend of polyphenols containing mostly hydroxytyrosol. Hydroxytyrosol $[5,17]$ is a strong inhibitor of metal-induced oxidation of low density lipoprotein. Furthermore, metal-independent oxidation is also significantly counteracted by hydroxytyrosol. The antioxidant activity of hydroxytyrosol, which has been shown to be more effective than butylated hydroxytoluene or vitamin $\mathrm{E}$, was also confirmed by the use of stable free radicals. Likewise, hydroxytyrosol is a scavenger of superoxide anions. In addition, a scavenging effect of hydroxytyrosol was demonstrated with respect to potent oxidants as the hypochlorous acid, produced during inflammatory processes (reviewed by Visioli and Bernardini [5]). Antioxidant activities have also been shown against DNA damage, hydrogen peroxide-induced insult to red blood cells. Quite interestingly, hydroxytyrosol maintains antioxidant activities also in vivo [18]. It protects from second

Table 3

Serum Hydroxytyrosol and its metabolites

\begin{tabular}{lllll}
\hline Groups & $\begin{array}{l}\text { T } \\
(\mathrm{ng} / \mathrm{mL})\end{array}$ & $\begin{array}{l}\text { HT (DOPET) } \\
(\mathrm{ng} / \mathrm{mL})\end{array}$ & $\begin{array}{l}\text { HTS1 } \\
(\mathrm{ng} / \mathrm{mL})\end{array}$ & $\begin{array}{l}\text { HTS2 } \\
(\mathrm{ng} / \mathrm{mL})\end{array}$ \\
\hline Ctrl & n.d. & $<1.0$ & n.d. & n.d. \\
EtOH & n.d. & $<1.0$ & n.d. & n.d. \\
Poly & n.d. & $<1.0$ & $11 \pm 1$ & $9 \pm 2$ \\
EtOH+Poly & n.d. & $<1.0$ & $23.4 \pm 0.5$ & $22.0 \pm 0.4$ \\
\hline
\end{tabular}

HT(DOPET), hydroxytyrosol; HTS1, hydroxytyrosol sulfate 1; HTS2, hydroxytyrosol sulfate 2; n.d., not detected; T, tyrosol hand smoke-induced oxidation [19], ameliorates lipid profile and decreases atherosclerosis development [20]. Some of these experiments have been performed with blends of olive phenols, in which hydroxytyrosol was the most important ingredient in concentration but not the exclusive one (as in the present study). Hence, a synergy with other olive phenols is highly possible. It was also shown that olive polyphenols in male alcoholics may modulate serum brain-derived neurotrophic factor during withdrawal [14].

Our findings show that olive polyphenol supplementation determines the appearance in the serum of hydroxytyrosol metabolites (i.e., hydroxytyrosol sulphate 1 and hydroxytyrosol sulphate 2), demonstrating that olive polyphenols are absorbed in the mouse gastrointestinal tract. Basal hydroxytyrosol was detected in the serum of all groups of mice regardless polyphenol's administration. The hydroxytyrosol is not only the main phenolic compound found in olives and olive products but it can also be produced endogenously [13] as a product of dopamine oxidative metabolism known as DOPET (3,4-dihydroxyphenylethanol) [21]. It has been also shown that alcohol intake might lead to an increase in DOPET due to the interaction between ethanol and the dopamine oxidative metabolism [22,23]. Furthermore, the hydroxytyrosol bioavailability depends on an extensive metabolic pathway producing different metabolites as the glucuronide and sulfates conjugates. Another minor metabolic pathway is the conversion of hydroxytyrosol into homovanillyl alcohol catalyzed by COMT (catechol-O-methyltransferase) [21].

Our results provide evidence that in the serum of Ctrl mice and $\mathrm{EtOH}$ mice no hydroxytyrosol metabolites were detected while both isomers of hydroxytyrosol sulfates (HTS1 and HTS2) were observed in the serum of Poly and Poly $+E t O H$ mice with highest levels in the Poly $+E T O H$ group, thus confirming the enhancement of hydroxytyrosol metabolism [24] as an effect of ethanol administration. Furthermore, both in physiological condition (Ctrl) and in oxidative stress situation (due to alcohol intake) we did not observe hydroxytyrosol metabolites. We do believe that, possibly due to the low levels of endogenous hydroxytyrosol, the ethanol administration did not potentiate the dopamine oxidative metabolism. However, hydroxytyrosol metabolites were detected in the serum of polyphenolsadministered mice. Quite interestingly, the concomitant presence of polyphenols and ethanol appears to counteract the $\mathrm{EtOH}$-induced oxidative effect as indicated by the reduction of FORT serum level. Moreover, the larger presence of hydroxytyrosol metabolites following $\mathrm{EtOH}$ ingestion represents the first indication that the antioxidant effect of polyphenols could be significantly increased under EtOH-induced oxidative stress. The highest levels of sulphate metabolites of hydroxytyrosol in the Poly $+E t O H$ mice seems to be due to a greater efficacy and absorption of hydroxytyrosol as a potential inducer of cell defense responses to oxidative insult. Furthermore, there is evidence that nanomolar concentrations of polyphenols may elicit their actions acting as signaling molecules and through the upregulation of the endogenous antioxidant enzyme system (see Kim et al. [25] for review).

\section{Conclusions}

Although further investigations are necessary it may be speculated that, since both polyphenols and alcohol consumption are key factors of the Mediterranean diet and alcohol abuse is also a devastating problem in Western countries, the present study may represent a step forward in the attempt to 
disclose some biomolecular processes and behavioral responses underlying the antioxidant properties of polyphenols. These results may be of interest for studies in the fields of human nutrition and human addiction.

\section{References}

[1] Del Rio D, Costa LG, Lean ME, Crozier A. Polyphenols and health: What compounds are involved? Nutr Metab Cardiovasc Dis 2010;20:1-6.

[2] Manach C, Scalbert A, Morand C, Remesy C, Jimenez L. Polyphenols: Food sources and bioavailability. Am J Clin Nutr 2004;79:727-47.

[3] Biesalski HK. Polyphenols and inflammation: Basic interactions. Curr Opin Clin Nutr Metab Care 2007;10:724-8.

[4] Bhullar KS, Rupasinghe HP. Polyphenols: Multipotent therapeutic agents in neurodegenerative diseases. Oxid Med Cell Longev 2013;2013:891748.

[5] Visioli F, Bernardini E. Extra virgin olive oil's polyphenols: Biological activities. Curr Pharm Des 2011;17:786-804.

[6] Fiore M, Ceccanti M, Carito V, Tarani L, Ferraguti G, Chaldakov GN. Neurotrophins modulation by olive polyphenols. Curr Med Chem 2016;23:3189-97.

[7] Manzo-Avalos S, Saavedra-Molina A. Cellular and mitochondrial effects of alcohol consumption. Int J Environ Res Public Health 2010;7:4281-304.

[8] Espinet C, Gonzalo H, Fleitas C, Menal MJ, Egea J. Oxidative stress and neurodegenerative diseases: A neurotrophic approach. Curr Drug Targets 2015;16:20-30.

[9] Fiore M, Laviola G, Aloe L, di Fausto V, Mancinelli R, Ceccanti M. Early exposure to ethanol but not red wine at the same alcohol concentration induces behavioral and brain neurotrophin alterations in young and adult mice. Neurotoxicology 2009;30:59-71.

[10] De Nicoló S, Tarani L, Ceccanti M, Maldini M, Natella F, Vania A, et al. Effects of olive polyphenols administration on nerve growth factor and brain-derived neurotrophic factor in the mouse brain. Nutrition 2013;29:681-7.

[11] Cardinali A, Cicco N, Linsalata V, Minervini F, Pati S, Pieralice M, et al. Biological activity of high molecular weight phenolics from olive mill wastewater. J Agric Food Chem 2010;58:8585-90.

[12] Macchia T, Mancinelli R, Gentili S, Lugaresi EC, Raponi A, Taggi F. Ethanol in biological fluids: Headspace GC measurement. J Anal Toxicol 1995;19:241-6.

[13] Carito V, Ciafrè S, Tarani L, Ceccanti M, Natella F, Iannitelli A, et al. TNF-a and IL-10 modulation induced by polyphenols extracted by olive pomace in a mouse model of paw inflammation. Ann Ist Super Sanita 2015;51:382-6.

[14] Ceccanti M, Carito V, Vitali M, Iannuzzi S, Tarani L, De Nicoló S, et al. Serum BDNF and NGF modulation by olive polyphenols in alcoholics during withdrawal. J Alcoh Drug Depend 2015;3:214.

[15] Pavlatou MG, Papastamataki M, Apostolakou F, Papassotiriou I, Tentolouris N. FORT and FORD: Two simple and rapid assays in the evaluation of oxidative stress in patients with type 2 diabetes mellitus. Metabolism 2009;58:1657-62.

[16] Coban J, Oztezcan S, Dogru-Abbasoglu S, Bingul I, Yeșil-Mizrak K, Uysal M. Olive leaf extract decreases age-induced oxidative stress in major organs of aged rats. Geriatr Gerontol Int 2014;14:996-1002.

[17] Perez-Jimenez F, Alvarez de Cienfuegos G, Badimon L, Barja G, Battino M, Blanco A, et al. International conference on the healthy effect of virgin olive oil. Eur J Clin Invest 2005;35:421-4.

[18] Visioli F, Caruso D, Plasmati E, Patelli R, Mulinacci N, Romani A, et al. Hydroxytyrosol, as a component of olive mill waste water, is dosedependently absorbed and increases the antioxidant capacity of rat plasma. Free Radic Res 2001;34:301-5.

[19] Visioli F, Galli C, Plasmati E, Viappiani S, Hernandez A, Colombo C, et al. Olive phenol hydroxytyrosol prevents passive smoking-induced oxidative stress. Circulation 2000;102:2169-71.

[20] Gonzalez-Santiago M, Martin-Bautista E, Carrero JJ, Fonolla J, Baró L Bartolomé MV, et al. One-month administration of hydroxytyrosol, a phenolic antioxidant present in olive oil, to hyperlipemic rabbits improves blood lipid profile, antioxidant status and reduces atherosclerosis development. Atherosclerosis 2006;188:35-42.

[21] Rodriguez-Morato J, Xicota L, Fito M, Farre M, Dierssen M, de la Torre R. Potential role of olive oil phenolic compounds in the prevention of neurodegenerative diseases. Molecules 2015;20:4655-80.

[22] Davis VE, Walsh MJ, Yamanaka Y. Augmentation of alkaloid formation from dopamine by alcohol and acetaldehyde in vitro. J Pharmacol Exp Ther 1970;174:401-12.

[23] Schroder H, de la Torre R, Estruch R, Corella D, Martínez-González MA, Salas-Salvadó J, et al. Alcohol consumption is associated with high concentrations of urinary hydroxytyrosol. Am J Clin Nutr 2009;90:132935.

[24] Perez-Mana C, Farre M, Pujadas M, Mustata C, Menoyo E, Pastor A, et al. Ethanol induces hydroxytyrosol formation in humans. Pharmacol Res 2015;95-96:27-33.

[25] Kim HS, Quon MJ, Kim JA. New insights into the mechanisms of polyphenols beyond antioxidant properties; lessons from the green tea polyphenol, epigallocatechin 3-gallate. Redox Biol 2014;2:187-95. 